\title{
The effect of SARS-CoV-2 on the prescribing of antimicrobials and analgesics by NHS general dental practitioners in England
}

Nikolaus 0. A. Palmer ${ }^{\star 1}$ and Noha Seoudi²

\section{Key points}

There was an increase in antibiotic prescribing in NHS general dental practice during the SARS CoV-2 pandemic dental practice restrictions.
There was an increase in analgesic prescribing in NHS general dental practice during the SARS-CoV-2 pandemic dental practice restrictions.
There was inappropriate and increased prescribing of opioid analgesics in NHS general dental practice during the SARS-CoV-2 pandemic dental practice restrictions.

\begin{abstract}
Aims To ascertain the effect of SARS-CoV-2 on the utilisation of antibacterial agents and analgesics in primary dental care.
\end{abstract}

Methods Antibacterial agents and analgesics (eg paracetamol, aspirin) prescribed in England by general dental practitioners for the periods April-July 2019 and April-July 2020 were analysed.

Results Antibacterial agents prescribed during COVID-19 restrictions in $2020(799,282)$ were higher than a similar time period in $2019(654,332)$ by $22 \%$. Amoxicillin was used the most $(2020=65.0 \% ; 2019=66.3 \%)$ followed by metronidazole $(2020=30.2 \% ; 2019=28.7 \%)$. Erythromycin was prescribed at a similar rate, with lincosamides (clindamycin) prescribed more frequently in 2020 (2020 $=0.6 \% ; 2019=0.5 \%)$. Clarithromycin was prescribed twice more often in $2020(0.6 \%)$ in comparison to $2019(0.3 \%)$. Co-amoxiclav $(0.5 \%)$ and phenoxymethylpenicillin $(0.3 \%)$ were prescribed at a similar rate. Analgesics use increased by $84 \%(2020=28,563 ; 2019=15,507)$. Use of dihydrocodeine tartrate increased $(2020=40.9 \% ; 2019=32.9 \%)$, followed by diclofenac sodium $(2020=24.6 \%$; $2019=12.8 \%)$. The opposite trend was seen in relation to ibuprofen with use decreasing $(2020=19.4 \% ; 2019=39.8 \%)$ while paracetamol use only slightly increasing $(2020=15.1 \% ; 2019=14.6 \%)$.

Conclusions COVID-19 restrictions on dental care in England resulted in a marked increase in prescribing antibacterial agents and a very marked increase in prescription-only analgesics.

\section{Introduction}

A novel severe acute respiratory syndrome coronavirus 2 (SARS-CoV-2) causing respiratory symptoms was first identified in December 2019 following a cluster of atypical pneumonia cases in Wuhan, Hubei province, China. On 11 March 2020 , due to the alarming spread and severity, the

${ }^{1}$ General Dental Practitioner, Honorary Lecturer, University of Liverpool, UK: ${ }^{2}$ Senior Clinical Lecturer in Oral Microbiology, Centre for Oral Immunobiology and Regenerative Medicine, Barts and the London School of Medicine and Dentistry, Queen Mary University of London, London, E1 2AD, UK.

${ }^{\star}$ Correspondence to: Nikolaus Palmer

Email address: nikolaus.palmer@btinternet.com

Refereed Paper

Accepted 26 November 2020

https://doi.org/10.1038/s41415-020-2595-2
World Health Organisation declared coronavirus disease 2019 (COVID-19) a pandemic, with Europe the epicentre of the pandemic with more reported cases and deaths than the rest of the world combined. ${ }^{1}$

The disease is caused by one of the coronaviruses, which are a large family of viruses. Severe acute respiratory syndrome coronavirus (SARS-CoV), Middle East respiratory syndrome (MERS) and SARSCoV-2 are members of the same genera of the coronaviruses. They are composed of an envelope, a lipid layer and singlestranded large RNA. It was found that the genome sequence of SARS-CoV-2, the virus responsible for COVID-19, is $>90 \%$ identical to a bat coronavirus and belongs to lineage $\mathrm{B}$ of the betacoronaviruses. ${ }^{2}$
COVID-19 is transmitted from human to human, leading to the observed large-scale spread. ${ }^{3}$ SARS-CoV-2 can be passed from person to person by respiratory droplets, with some evidence suggesting transmission through contact and fomites. A recent systematic review suggested that some procedures were capable of generating aerosols associated with increased risk of SARS-CoV-2 transmission to healthcare workers (HCWs). ${ }^{4}$ Several scientists expressed concern at the lack of recognition of airborne transmission in aerosols, particularly in enclosed environments, and a requirement as part of infection prevention and control to mitigate this potential risk. ${ }^{5}$ It is well documented that dental handpieces and ultrasonic scalers cause the formation of splatter, droplets and aerosols 
contaminated with bacteria, viruses, fungi and blood. ${ }^{6,7}$

The SARS-CoV-2 viral load and virulence/ infective dose of aerosols produced during dental treatment is unknown at present. Within healthcare worldwide, it has been accepted that aerosol generating procedures (AGPs) are a potential risk of transmission, not only to HCWs but also subsequent patients and members of the public in the healthcare environment/surgery.

While patients with COVID-19 will have recognisable and easily screened symptoms, the level of asymptomatic carriers of the disease in the community is unknown. A recent systematic review and meta-analysis suggest that the proportion of asymptomatic cases was $20 \%$ (95\% CI; $17-25 \%, 79$ studies overall). ${ }^{8}$ In response to the challenge of the pandemic and known and potential transmissibility by provision of dental care, the National Health Service (NHS) - through the Office of the Chief Dental Officer (OCDO) and the health regulatory bodies of the General Dental Council and the Care Quality Commission - provided advice and guidance to protect and prevent transmission of SARS-CoV-2 to dentists, the dental team, patients and the wider population.

Lockdown with severe restrictions on movement of people was imposed by the UK government from 26 March 2020 and, alongside this announcement, the OCDO instructed dentists working in NHS primary care to stop all routine, non-urgent dental care including orthodontics. All dental practices were requested to establish, independently or in collaboration, remote urgent care services providing telephone triage for patients with urgent needs during normal working hours.

Management of urgent dental care in primary dental care was restricted to remote advice and remote prescribing of analgesia and antimicrobials, where appropriate. Where the patient's condition could not be managed due to severe pain, trauma or infection, they were to be referred to local urgent dental care systems (UDCs) established by collaboration between local dental care networks, commissioners and local dental committees. ${ }^{9}$ This also applied to patients with COVID-19 (diagnosed, symptomatic or living in the same household), shielded patients with a significant risk from COVID-19 or vulnerable patients. These patients referred to UDCs were assessed and managed on their individual merit and based on local UDC protocols, with prioritisation of emergency and urgent cases. By mid-April, it was reported that 130 out of 165 UDCs were open in addition to existing emergency and out-of-hours services. ${ }^{10}$ This increased to over 500 UDC sites before the resumption of dental services letter to NHS dental practices was published by the OCDO on 28 May 2020. ${ }^{11}$

Dental practices reopened from 8 June and were able to resume a safe and effective full range of care, with effective personal protective equipment and infection prevention and control, as recommended by Public Health England. Updated standard operating procedure guidance published by the OCDO for England on 28 August advised a continuation of remote consultations of all patients, providing advice, analgesics and antimicrobials, where appropriate. It also advised that, where face-to-face treatment was necessary, this should be focused on stabilising the patient's condition by minimising exposure risk to AGPs. ${ }^{12}$

Anecdotal evidence from dental social media sites suggests that, for the period during dental practice closure and remote triage, dental practitioners were inappropriately prescribing antimicrobial agents. In some cases, this appeared to be driven by an inability to access appropriate UDC management for drainage of infections and for acute pulpitis. Others reported cases where there was no clinical improvement following antimicrobial prescription and these patients were rejected by UDCs for definitive management, with a request for a further course or courses of antimicrobial agents, even where this was inappropriate.

It is well established in dental practice that acute pulpitis and dental infections are best managed by definitive treatment including removal of decay, extirpating the infected pulp, drainage of abscesses, or extractions where the prognosis for a tooth is poor. Antibiotics are not indicated for acute pulpitis or localised dental infections and should only be prescribed where there is evidence of a spreading infection. ${ }^{13,14}$ Analgesics for dental pain relief are advised/ prescribed following a comprehensive assessment of the patient's condition. They are indicated only as an adjunct to definitive treatment or where treatment must be delayed until the causative factors can be removed.

When prescribing an antimicrobial agent or analgesics for patients, NHS primary care dentists in England use an FP10D form, which is then dispensed by pharmacies within England. The NHS Business Services Authority
(NHSBSA) collects and publishes data on the number of items of each drug listed in the British National Formulary (BNF) dispensed by pharmacies in England. These data are published annually as the prescription cost analysis. ${ }^{15}$

A recently published study indicated increased dental antibiotic prescribing in all regions in England. The highest rate was seen in the East of England during June and the lowest rate was in London during April. London showed the highest increase in July, with $60 \%$ more antibiotic used in July than April 2020. ${ }^{16}$ This study did not analyse the analgesics used or the type of antibiotic agent prescribed in the period of COVID-19 dental care restrictions.

The aim of this study is to investigate the effect of the COVID-19 pandemic on antibiotic and analgesic prescribing by NHS primary care dentists during the period of lockdown and the severe restrictions placed on management of patients with dental pain and/or infection.

\section{Methodology}

A Freedom of Information request (10334) for prescribing data from NHSBSA for two corresponding time periods to compare antimicrobial agents and analgesics prescribing by NHS dentists was as follows:

- The total number of items of antibacterial agents in the BNF section (0501) prescribed and dispensed in England by general dental practitioners (GDPs) for the period 1 April-1 July 2019 expressed as individual drug names, chemical names and number of items

- The total number of items of antibacterial agents in the BNF section (0501) prescribed and dispensed in England by GDPs for the period 1 April-1 July 2020 expressed as individual drug names, chemical names and number of items

- The total number of items of analgesics (paracetamol, aspirin, dihydrocodeine, diclofenac sodium and ibuprofen) prescribed by NHS GDPs for the periods 1 April-1 July 2019 and 1 April-1 July 2020.

All the data received were entered into Prism 8 statistics pack (GraphPad, CA, USA) and analysed for frequencies of all variables. Two-way ANOVA with Bonferroni multiple comparisons tests were used to test for significant differences between the two time periods. 


\section{Results}

Antibacterial prescribing in the period between April-June 2019

The total number of antibacterial agent items prescribed by NHS GDPs in the period between April-June 2019 was 654,332. As shown in Table 1, amoxicillin accounted towards $66.3 \%$ of the total antibacterial agents prescribed followed by metronidazole (28.7\%), erythromycin $(2.4 \%)$, clindamycin $(0.5 \%)$, co-amoxiclav $(0.5 \%)$, phenoxymethylpenicillin $(0.3 \%)$ and clarithromycin $(0.3 \%)$.

Antibacterial prescribing in the period between April-June 2020

The total antibacterial agent items prescribed by NHS GDPs in the period between AprilJune 2020 was 799,282. Amoxicillin accounted towards $65.0 \%$ of the total antibacterial agents prescribed followed by metronidazole (30.2\%), erythromycin $(2.2 \%)$, clindamycin $(0.6 \%)$, clarithromycin $(0.6 \%)$, co-amoxiclav $(0.5 \%)$ and erythromycin ethylsuccinate $(0.2 \%)$, as shown in Table 2.

\section{Antibacterial prescribing analytical statistics}

The total items prescribed in the period between April-June $2020(799,282)$ was higher than the same period in $2019(654,332)$ by $22 \%$. However, this increase was not statistically significant ( $p>0.05)$. Amoxicillin accounted for $65.0 \%$ in 2020 and $66.3 \%$ in 2019 , followed by metronidazole which accounted for $30.2 \%$ in 2020 in comparison to $28.7 \%$ in 2019.

Erythromycin was used in a similar rate in both years $(2.2 \%$ in 2020 in comparison to $2.4 \%$ in 2019 [ $p>0.05]$ ]). Interestingly, there was a trend towards prescribing lincosamides (clindamycin) more frequently in the period between April-June 2020 in comparison to the same period in $2019(0.6 \%$ and $0.5 \%$, respectively). Clarithromycin was used twice as frequently in 2020 (0.6\%) in comparison to 2019 (0.3\%). However, this difference did not reach statistical significance $(\mathrm{p}<0.5)$. Co-amoxiclav and phenoxymethylpenicillin were prescribed at the same rate in 2020 in comparison to 2019 ( $0.5 \%$ and $0.3 \%$, respectively).

\section{Analgesics prescribed in the period} between April-June 2019

Total analgesics prescribed in the period between April-June 2019 was 15,507. Ibuprofen accounted for $39.8 \%$ of the total analgesics prescribed followed by dihydrocodeine
Table 1 Antibacterial agents prescribed during the period between April-June 2019

\begin{tabular}{l|l|l}
\multirow{2}{*}{ Antibacterial agents } & \multicolumn{2}{c}{ April-June 2019} \\
\cline { 2 - 3 } & Total (n) & (\%) \\
\hline Oxytetracycline & 92 & $<0.01$ \\
\hline Tetracycline & 141 & $<0.01$ \\
\hline Cefradine & 147 & $<0.01$ \\
\hline Azithromycin & 591 & 0.09 \\
\hline Cefalexin & 1,012 & 0.15 \\
\hline Doxycycline hyclate & 1,047 & 0.16 \\
\hline Erythromycin ethylsuccinate & 1,816 & 0.27 \\
\hline Erythromycin stearate & 1,889 & 0.28 \\
\hline Clarithromycin & 2,079 & 0.31 \\
\hline Phenoxymethylpenicillin (penicillin V) & 2,263 & 0.34 \\
\hline Co-amoxiclav (amoxicillin/clavulanic acid) & 2,948 & 0.45 \\
\hline Clindamycin hydrochloride & 3,290 & 0.50 \\
\hline Erythromycin & 15,709 & 2.40 \\
\hline Metronidazole & 187,751 & 28.69 \\
\hline Amoxicillin & 433,557 & 66.25 \\
\hline Total & 654,332 & 100.00 \\
\hline & & \\
\hline
\end{tabular}

Table 2 Antibacterial agents prescribed in the period April-June 2020

\begin{tabular}{ll|l}
\multirow{2}{*}{ Antibacterial agents } & \multicolumn{2}{c}{ April-June 2020 } \\
\cline { 2 - 3 } Oxytetracycline & Total (n) & $(\%)$ \\
\hline Cefradine & 79 & $<0.01$ \\
\hline Tetracycline & 125 & $<0.01$ \\
\hline Azithromycin & 136 & $<0.01$ \\
\hline Doxycycline hyclate & 439 & 0.05 \\
\hline Cefalexin & 779 & 0.09 \\
\hline Phenoxymethylpenicillin (Penicillin V) & 823 & 0.10 \\
\hline Erythromycin stearate & 1,458 & 0.18 \\
\hline Erythromycin ethylsuccinate & 1,546 & 0.19 \\
\hline Co-amoxiclav (Amoxicillin/clavulanic acid) & 1,926 & 0.24 \\
\hline Clarithromycin & 3,630 & 0.45 \\
\hline Clindamycin hydrochloride & 4,487 & 0.56 \\
\hline Erythromycin & 4,807 & 0.60 \\
\hline Metronidazole & 17,805 & 2.21 \\
\hline Amoxicillin & 241,357 & 30.20 \\
\hline Total & 519,885 & 65.10 \\
\hline & 799,282 & 100.00 \\
\hline
\end{tabular}


(32.9\%), paracetamol (14.6\%) and diclofenac sodium $(12.8 \%)$, as shown in Table 3.

\section{Analgesics prescribed in the period between April-June 2020}

Total analgesics prescribed in the period between April-June 2020 was 28,563. Dihydrocodeine tartrate accounted for $40.9 \%$ of the total analgesics prescribed followed by diclofenac sodium (24.6\%), ibuprofen (19.4\%) and paracetamol (15.1\%), as shown in Table 4 .

\section{Analgesics prescribing analytical statistics}

The total analgesics prescribed in the period between April-June 2020 was $84 \%$ higher than that of the same period in 2019. However, this difference did not reach statistical significance ( $p>0.05)$. Dihydrocodeine and diclofenac were prescribed at a higher rate in 2020 when compared with the same period in 2019 . Dihydrocodeine tartrate accounted for $40.9 \%$ in 2020 and $32.9 \%$ in 2019 of the total analgesics used, while diclofenac sodium accounted for $24.6 \%$ in 2020 and $12.8 \%$ in 2019.

Interestingly, the opposite trend was seen in relation to ibuprofen, with ibuprofen accounting for $19.4 \%$ of the total analgesics prescribed by GDPs in the COVID-19 restriction period of 2020 in comparison to $39.8 \%$ in the same period in 2019, while paracetamol accounted for $15.1 \%$ of the total in 2020 and $14.6 \%$ in 2019. However, this difference did not reach statistical significance $(\mathrm{p}<0.5)$.

\section{Discussion}

Since March 2020, the dental profession has focused on protecting and preventing the transmission of SARS-CoV-2 to their team, patients and the wider population due to the mortality (globally estimated at present as $>1.9$ million) and morbidity associated with COVID-19. The severe restrictions placed on NHS GDPs delivering routine and emergency care prevented face-to-face contact and clinical patient management for a period of three months. Patient care was limited to remote consultation, assessment of the patient's problem, advice and prescribing of analgesics and/or antimicrobial agents, or in severe cases, referral for definitive treatment.

This study attempted to measure the effect of restrictions on provision of patient care in relation to the management of pain and dental infections by antimicrobial agents and analgesics. No data were available from solely private practices

Table 3 Analgesics prescribed in the period between April-June 2019

\begin{tabular}{l|l|l}
\multirow{2}{*}{ Analgesics } & \multicolumn{2}{|c}{ April-June 2019} \\
\cline { 2 - 3 } & Total $(\mathbf{n})$ & $(\%)$ \\
\hline Aspirin & $*$ & $*$ \\
\hline Diclofenac sodium & 1,980 & 12.77 \\
\hline Paracetamol & 2,258 & 14.56 \\
\hline Dihydrocodeine tartrate & 5,104 & 32.91 \\
\hline Ibuprofen & 6,165 & 39.76 \\
\hline Total & $\mathbf{1 5 , 5 0 7}$ & $\mathbf{1 0 0 . 0 0}$ \\
\hline $\begin{array}{l}\text { Key: } \\
*=\end{array}$ less than five prescriptions; therefore, data withheld by information commissioner to protect personal data
\end{tabular}

Table 4 Analgesics prescribed in the period April-June 2020

\begin{tabular}{l|l|l|}
\hline \multirow{2}{*}{ Analgesics } & \multicolumn{3}{|c}{ April-June 2020} \\
\cline { 2 - 3 } & Total (n) & (\%) \\
\hline Aspirin & $*$ & $*$ \\
\hline Paracetamol & 4,310 & 15.09 \\
\hline Ibuprofen & 5,534 & 19.37 \\
\hline Diclofenac sodium & 7,036 & 24.63 \\
\hline Dihydrocodeine tartrate & 11,683 & 40.91 \\
\hline Total & $\mathbf{2 8 , 5 6 3}$ & $\mathbf{1 0 0 . 0 0}$ \\
\hline $\begin{array}{l}\text { Key: } \\
*\end{array}=$ less than five prescriptions; therefore, data withheld by information commissioner to protect personal data
\end{tabular}

(NHS prescription forms cannot be used for patients undergoing private treatment) or from secondary care providers. The study was limited to NHS primary care dental practice as verified up-to-date prescription data were available from the NHSBSA.

The study was also limited in that the authors had no indication of the clinical signs and symptoms primary care dental practitioners were treating with antimicrobial agents and/or analgesics remotely. Also, it was not possible to determine whether more than one course or multiple courses were required to alleviate individual patients' symptoms in the absence of definitive treatment. Furthermore, information on dose, frequency and duration of the different antibiotics prescribed were not available to analyse.

While it was not possible to assess the appropriateness of the prescribing during the period of remote diagnosis and management, it is clear that the number of prescriptions for both analgesics and antimicrobials had increased substantially when compared to an equivalent period pre-COVID-19. This is more concerning when the total number of NHS GDPs remained around 24,684 in the period between April-June 2019 and April-June 2020 (NHS Digital data). It is estimated that $4 \%$ less adult patients and $17 \%$ less children were seen in 2020 when compared with the corresponding period in 2019. ${ }^{17}$

Antimicrobial resistance has increased as a result of widespread use of antimicrobial agents over many years and the inappropriate prescribing of antimicrobial agents by healthcare professions remains a concern. It is recognised that, as antimicrobial agents become less effective, the healthcare gains (through, for example, organ/stem cell transplants, major surgery, chemotherapy) will be lost, leaving these patients with a higher risk of mortality. Unfortunately, antimicrobial resistance has been, and will be in the long term, a greater worldwide problem and major threat to public health than COVID-19. It has been estimated that 700,000 people die of antibiotic-resistant infections every year and that, by 2050 , the global cost will be ten million lives and $£ 69$ trillion a year. ${ }^{18}$ 
Antimicrobials prescribed during COVID-19 dental practice restrictions

The majority of the antimicrobial agents prescribed were either amoxicillin or metronidazole, which are indicated as the first choices for dental infections ${ }^{13,14}$ where there is evidence of systemic involvement, and they are used as an adjunct to definitive treatment such as extraction, root canal treatment or surgical drainage. It is accepted that there are occasions when definitive treatment has to be delayed and antimicrobial agents are necessary to alleviate/prevent a worsening of symptoms. These patients should, however, be treated definitively as soon as possible to avoid repeat prescribing of antimicrobials. ${ }^{13}$

Of note, in the period between April-June 2020 , clindamycin was prescribed $10 \%$ more $(4,807$ prescriptions) than it was in the same period in 2019 (3,290 prescriptions). While the level of prescribing during practice restrictions was small, there is no evidence to suggest that there is an increased rate of allergic reaction to penicillin or a reported increased rate of severe odontogenic infection to justify the rise in the prescribing of clindamycin. Even though clindamycin has good bone penetration and a broad antibacterial spectrum, including good anaerobic activity, it has a marked side effects profile, including dysbiosis of the gut microflora resulting in the reported increasing incidence of Clostridium difficile. Clindamycin should therefore only be reserved for cases of severe odontogenic infection in patients who are allergic to penicillin. ${ }^{13}$ Clearly, these cases should have been managed in a secondary care setting, receiving prompt appropriate dental management in addition to the antimicrobial agent.

Clarithromycin use in dentistry is normally limited to patients with hypersensitivity to penicillin or patients who have had a recent course of penicillin. ${ }^{13}$ Clarithromycin is better tolerated than erythromycin, which broadly remained constant between the two periods. In the period between April-June 2020, the prescribing of clarithromycin doubled $(4,487$ prescriptions) in comparison to its use in the same period in 2019 (2,079 prescriptions). There is no evidence to suggest that there is an increased rate of allergic reaction to penicillin in the literature. It is possible that, in the absence of options for definitive treatment during restrictions on general dental practice, it might have been the antibiotic of choice where treatment had to be delayed and penicillin and metronidazole had been prescribed previously. Unfortunately, lack of individual patient data on the indications for prescribing preclude judgements on appropriateness of the prescription.

\section{Analgesics prescribed during COVID-19 dental practice restrictions}

The prescribing of analgesics to control dental pain and fever as an adjunct to definitive treatment is well established within dental practice. When prescribing or advising pain relief for acute dental pain, dentists should consider the medication's effectiveness but also the potential to cause harm. Guidelines and advice on prescribing analgesia for mildto-moderate dental pain recommend the use of paracetamol or ibuprofen either alone or in combination. ${ }^{19,20}$ It is likely that more paracetamol and ibuprofen were prescribed or advised in both time periods in this study for dental pain as these are more cheaply available over the counter (OTC).

Diclofenac and dihydrocodeine are prescription-only drugs, not available OTC, with dihydrocodeine a Schedule 5 controlled drug. Opioid analgesics, including dihydrocodeine, are generally prescribed for acute and chronic pain but are relatively ineffective for dental pain. In an overview of systematic reviews, evaluating the efficacy or reported adverse events associated with orally administered analgesics for relief of acute dental pain, it was found that a combination of ibuprofen and paracetamol was superior in the magnitude of dental pain relief to any opioid-containing medication. ${ }^{21}$ The additional benefit from using dihydrocodeine or diclofenac sodium in comparison to ibuprofen or paracetamol is questionable. The Oxford League Table of Analgesic Efficacy states that the number needed to treat is 1.7 for ibuprofen 600/800, 1.8 for diclofenac 100, 2.2 for paracetamol $1,000+$ codeine 60 , 2.5 for ibuprofen $400,2.6$ for diclofenac 25 , 2.7 for diclofenac 50, 2.7 for ibuprofen 200, 3.5 for paracetamol 500, 4.2 for paracetamol $600 / 650+$ codeine 60 and 16.7 for codeine $60 .{ }^{22}$

The level of prescribing of dihydrocodeine, both before COVID-19 and during the period of COVID-19 restrictions on dental practice, is a cause for concern due to the potential for drug addiction and abuse. NHS dental prescribing of dihydrocodeine tartrate in the period of April-June 2020 was 24\% higher (11,683 prescriptions) than prescribed in the same period in 2019 (5,104 prescriptions). Due to its high profile of side effects - including constipation, dizziness, drowsiness, fatigue, hot flushes, increasing sweating, nausea, pruritus and vomiting - and the lack of antiinflammatory effect, they should be reserved for when the clinical benefit of its use outweighs the possible side effects. ${ }^{23}$

It is possible that NHS dental practitioners, during the period of no face-to-face definitive treatment of acute dental pain (for example, excavation of caries and obtundent dressing, extraction, root canal therapy), felt the need to prescribe opiate analgesics, either due to the delay in definitive management or lack of emergency provision through the UDCs. Qualitative research is urgently required to assess GDPs' reasons for prescribing opiate analgesics.

Diclofenac is one of the non-steroidal antiinflammatory drugs (NSAIDs) which is used in the treatment of acute and chronic severe pain. It has analgesic, anti-inflammatory and antipyretic effects, but it also has known side effects such as NSAID-induced asthma, chronic renal dysfunction and gastrointestinal enteropathy, including small and large bowel ulceration. ${ }^{24,25}$ The use of diclofenac sodium increased markedly during the period between April-June 2020 (7,036 prescriptions) in comparison to the same period in 2019 (1,980 prescriptions). In the same period, there was a $10 \%$ increase in prescriptions of clindamycin, and it is worth noting that the combined use of diclofenac and clindamycin can lead to serious complications such as colon perforation with acute peritonitis. ${ }^{26}$

No data are available to clarify why GDPs felt the need to prescribe diclofenac in preference to paracetamol and/or ibuprofen. It is possible that GDPs prescribed diclofenac when remotely faced with patients reporting severe acute dental pain which was not responding to OTC analgesics in the absence of definitive clinical treatment.

\section{Conclusions}

COVID-19 restrictions on the provision of NHS primary dental care in England during AprilJune 2020 resulted in a marked increase in prescribing antibacterial agents. Antimicrobial stewardship needs to be re-embedded in dental practice to reduce the further development of antimicrobial resistance.

A very marked increase in prescribing of dihydrocodeine and diclofenac analgesics was also observed during the COVID-19 restrictions. The prescribing of dihydrocodeine 
and diclofenac for acute dental pain needs to be investigated because of the large side effects profile of these analgesics and lack of added benefit of their use in comparison to ibuprofen and paracetamol.

\section{Learning points}

During the period of the SARS-CoV-2 pandemic NHS general dental practice restrictions, there was:

1. Increased NHS antibiotic prescribing

2. Increased NHS analgesic prescribing

3. Inappropriate and increased NHS prescribing of opioid analgesics.

The above three points need to be addressed and audited to prevent future deleterious effects.

GDPs are urged to follow the rules of antimicrobial stewardship when prescribing antibiotics. The Public Health England dental antimicrobial stewardship toolkit is available at https://www.gov.uk/guidance/ dental-antimicrobial-stewardship-toolkit.

Guidelines for antimicrobial prescribing in dentistry are available at https://www.fgdp.org. uk/antimicrobial-prescribing and https://bnf. nice.org.uk, and in Scotland at https://www. sapg.scot/media/5473/statement-on-pen-v-indental-infections.pdf and https://www.sdcep. org.uk/published-guidance/drug-prescribing/.

\section{Conflict of interest}

Authors declare no conflict of interest.

\section{Acknowledgments}

Authors acknowledge the help of the NHS Business

Services Authority (NHSBSA) in providing the

required data for this study.

\section{References}

1. World Health Organisation. WHO Director-General's opening remarks at the media briefing on COVID-19. 2020. Available at https://www.who.int/dg/speeches/ detail/who-director-general-s-opening-remarks-atthe-media-briefing-on-covid-19---11-march-2020 (accessed October 2020).

2. Zhu N, Zhang D, Wang W et al. A Novel Coronavirus from patients with pneumonia in China 2019. N Engl J Med 2020; 382: 727-733.

3. Chan FY, Yuan S, Kok K H et al. A familial cluster of pneumonia associated with the 2019 novel coronavirus indicating person-to-person transmission: a study of a family cluster. Lancet 2020; 395: 514-523.

4. Tran K, Cimon K, Severn M et al. Aerosol Generating Procedures and Risk of Transmission of Acute Respiratory Infections to Healthcare Workers: A Systematic Review. PLoS One 2012; DOI: 10.1371/ journal.pone.0035797.

5. Morawska L, Milton D K. It is time to address airborne transmission of COVID-19. Clin Infect Dis 2020; DOI: 10.1093/cid/ciaa939.

6. Harrel S, Molinari J A. Brief review of the literature and infection control implications. J Am Dent Assoc 2004: 135: 429-437.

7. Kobza J, Pastuszka J S, Bragoszewska E. Do exposures to aerosols pose a risk to dental professionals? Occup Med (Lond) 2018; 68: 454-458.

8. Buitrago-Garcia D, Egli-Gany D, Counotte M J et al. Occurrence and transmission potential of asymptomatic and presymptomatic SARS-CoV-2 infections: A living systematic review and meta-analysis. PLoS Med 2020; DOI: $10.1371 /$ journal.pmed.1003346.

9. NHS England. COVID-19 guidance and standard operating procedure: for the provision of urgent dental care in primary care dental settings and designated urgent dental care settings. 2020. Available at https:// www.england.nhs.uk/coronavirus/wp-content/uploads/ sites/52/2020/06/C0581-covid-19-urgent-dental-caresop-update-16-june-20-.pdf (accessed October 2020).

10. Rapson J. 'People in pain have nowhere to go' as NHSE 'drags its heels' on urgent care system. 2020. Available at https://www.hsj.co.uk/free-for-non-subscribers/ people-in-pain-have-nowhere-to-go-as-nhse-dragsits-heels-on-urgent-care-system/7027427.article (accessed December 2020).

11. Hurley S, Neligan M. Resumption of dental services in England. 2020. Available at https://www. england.nhs.uk/coronavirus/wp-content/uploads/ sites/52/2020/03/Urgent-dental-care-letter-28-May. pdf (accessed October 2020).

12. Office of Chief Dental Officer England. Standard operating procedure: transition to recovery (28 August 2020). 2020. Available at https://www.england.nhs.uk/ coronavirus/wp-content/uploads/sites/52/2020/06/ C0575-dental-transition-to-recovery-SOP-4June.pdf (accessed December 2020).
13. Faculty of General Dental Practice (UK). Antimicrobial prescribing guidelines for general dental practitioners. 2016. Available online at https://www.fgdp.org.uk/ guidance-standards/antimicrobial-prescribing-gdps (accessed October 2020).

14. SDCEP. Drug prescribing for dentistry. 2016. Available online at https://www.sdcep.org.uk/publishedguidance/drug-prescribing/ (accessed October 2020).

15. NHS Business Services Authority. Prescription Cost Analysis - England 2019. 2020. Available at https://www.nhsbsa.nhs.uk/statistical-collections/ prescription-cost-analysis-england/prescription-costanalysis-england-2019 (accessed October 2020).

16. Shah S, Wordley V, Thompson W. How did COVID-19 impact on dental antibiotic prescribing across England? Br Dent J 2020; 229: 601-604.

17. NHS Digital. NHS Dental Statistics for England - 201920: Annual Report. 2020. Available at https://digital. nhs.uk/data-and-information/publications/statistical/ nhs-dental-statistics/2019-20-annual-report/spotlightreport (accessed October 2020).

18. Review on Antimicrobial Resistance. Tackling drug-resistant infections globally: Final report and recommendations. 2016. Available at https://amrreview.org/sites/default/files/160518_Final\%20 paper_with\%20cover.pdf (accessed October 2020).

19. National Institute for Health and Care Excellence. Analgesia - mild-to-moderate pain. 2020. Available online at https://cks.nice.org.uk/topics/analgesia-mildto-moderate-pain/ (accessed October 2020).

20. Public Health England. National analgesic advice sheet for dentists. 2016. Available online at https://www.gov. uk/guidance/dental-antimicrobial-stewardship-toolkit (accessed October 2020).

21. Moore PA, Zeigler K M, Lipman R D, Aminoshariae A, Carrasco-Labra A, Mariotti A. Benefits and harms associated with analgesic medications used in the management of acute dental pain. An overview of systematic reviews. J Am Dent Assoc 2018; DOI: 10.1016/j.adaj.2018.02.012.

22. Bandolier. The Oxford League Table of Analgesic Efficacy. 2007. Available at http://www.bandolier. org.uk/booth/painpag/Acutrev/Analgesics/lftab.html (accessed October 2020).

23. DePriest A Z, Puet B L, Holt A C, Roberts A, Cone E J. Metabolism and disposition of prescription Opioids: a review. Forensic Sci Rev 2015; 27: 115-145.

24. Byrne M F, McGuiness J, Smyth C M et al. Nonsteroidal antiinflammatory drug-induced diaphragms and ulceration in the colon. Eur J Gastroenterol Hepatol 2002; 14: 1265-1269.

25. Haas D A. An update on analgesics for the management of acute postoperative dental pain. J Can Dent Assoc 2002; 68: 476-482.

26. Ervens J, Schiffmann L, Berger G, Hoffmeister B. Colon perforation with acute peritonitis after taking clindamycin and diclofenac following wisdom tooth removal. J Craniomaxillofac Surg 2004; 32: 330-334. 